\title{
Postoperative Pain Relief Using Wound Infiltration With 0.5\% Bupivacaine in Single-Incision Laparoscopic Surgery for an Appendectomy
}

\author{
So Ra Ahn, Dong Baek Kang, Cheol Lee ${ }^{1}$, Won Cheol Park, Jeong Kyun Lee \\ Departments of Surgery and ${ }^{1}$ Anesthesiology, Digestive Disease Research Institute and Institute of Medical Science, Wonkwang University \\ College of Medicine, Iksan, Korea
}

Purpose: Recently, single-incision laparoscopic surgery (SILS) has been popular for minimally invasive surgery and cosmetic improvement. However, some papers have reported that SILS for an appendectomy (SILS-A) has had the more postoperative complaints of pain. We investigated postoperative pain relief using wound infiltration with $0.5 \%$ bupivacaine in SILS-A and compared the result with that for conventional SILS-A.

Methods: Between July 2010 and September 2012, 75 patients who underwent SILS-A were enrolled in this study. The patients were randomly assigned to two groups: conventional SILS-A group (C-SILS-A) or wound infiltrated with $0.5 \%$ bupivacaine in SILS-A group (W-SILS-A). Forty-five patients were in the C-SILS-A, and 30 patients were in the W-SILS-A. Patients with perforated appendicitis were excluded. The clinical outcomes were compared between the groups by using the verbal numerical rating scale (VNRS).

Results: Clinical outcomes were similar in both study groups except for the pain score. The W-SILS-A group showed significantly lower numbers of additional pain killers and lower VNRS scores 1, 6, and 12 hours after surgery than the CSILS-A group.

Conclusion: W-SILS-A is a technically simple and effective method of reducing early postoperative pain. It may be applicable in SILS-A for pain control system.

Keywords: Bupivacaine; Laparoscopy; Postoperative pain; Appendectomy

\section{INTRODUCTION}

Acute appendicitis is the most common disease requiring emergency surgery. During the era of laparoscopic surgery, a common trend has been towards less invasive techniques, and a natural extension of the trend has been to perform operations without scarring. In numerous studies, when the conventional laparoscopic

Received: June 12, 2013 - Accepted: October 3, 2013

Correspondence to: Dong Baek Kang, M.D.

Department of Surgery, Digestive Disease Research Institute and Institute of Medical Science, Wonkwang University College of Medicine,

460 Iksan-daero, Iksan 570-974, Korea

Tel: +82-63-859-1499, Fax: +82-63-855-2386

E-mail: east1st@wku.ac.kr

(c) 2013 The Korean Society of Coloproctology

This is an open-access article distributed under the terms of the Creative Commons Attribution NonCommercial License (http://creativecommons.org/licenses/by-nc/3.0) which permits unrestricted noncommercial use, distribution, and reproduction in any medium, provided the original work is properly cited. appendectomy using 3 ports (C-LA) compared with open appendectomy, it has advantages of reduced pain, reduced hospital stay, and enhanced cosmetic effects $[1,2]$.

Recently, as technology and innovation continue to advance the field of minimally-invasive surgery, single-incision laparoscopic surgery (SILS) is being applied to diverse surgeries as a new technique for minimally invasive surgery [3-8]. In studies comparing SILS for an appendectomy (SILS-A) with a C-LA in adults, the former was superior from the cosmetic viewpoint, and the incidence of complications was not different. Thus, recently, it was reported as a technique that could be performed safely in adults [913]. However, some papers reported that SILS-A had more postoperative complaints of pain [14-16].

The administration of local anesthetics into the wound before the incision (preemptive analgesia) has been demonstrated to reduce postoperative pain in many kinds of surgeries such as inguinal herniorrhaphy, cholecystectomy, tonsillectomy, diagnostic 
laparoscopic procedures, gynecological procedures and some orthopedic procedures [17-22]. Bupivacaine is often administered by epidural injection during spinal anesthesia. It is also commonly injected into surgical wound sites for the relief of postoperative pain [23]. Therefore, we investigated postoperative pain when using wound infiltration with $0.5 \%$ bupivacaine in SILS-A and compared it with that in conventional SILS-A.

\section{METHODS}

This study was a retrospective review of medical records, and the technique of the SILS-A was approved by the Ethical Committee of Wonkwang University Hospital. This study was performed on 75 patients with appendicitis who underwent either a conventional SILS for appendectomy (C-SILS-A) or wound infiltrated with $0.5 \%$ bupivacaine in SILS-A (W-SILS-A) by the same surgeon from July 2010 to September 2012 at our hospital. Forty-five patients received a C-SILS-A, and 30 received a W-SILS-A. Patients with a perforated appendicitis were excluded.

Prior to surgery, abdominal ultrasonography or computed tomography was performed on all patients. In regard to surgical methods, the C-LA, SILS-A, and laparotomy were explained to the patients, after which the method was selected by the patients themselves or their guardians. General anesthesia was administered to all patients. Simultaneously with the diagnosis of appendicitis, an antibiotic, second generation cephalosporin, was administered. In the supine position, the surgeon stood in the left lower area of the patient, leaning toward the lower extremities, and the first assistant manipulated the laparoscope on the right upper side of the surgeon.

C-SILS-A was performed in the supine position under general anesthesia, and in the umbilical area, according to the open incision method, a 1.5- to 2-cm vertical incision was made. If the umbilical area was severely dirty or malodorous, avoiding the center of the umbilical area, in the area above the umbilical area or based on the umbilical area, a half-moon incision window, $1.5-$ to $2-\mathrm{cm}$ in size, was made. The Octoport wound retractor (Dalim, Gangseo, Seoul, Korea) was put into the peritoneal cavity covering from the skin to the peritoneum. A $30^{\circ}, 5-\mathrm{mm}$ laparoscopic camera (Karl-Storz, Tuttlingen, Germany) was used, and the mesoappendix was ligated and dissected by the application of the LigaSure (Valleylab, Boulder, CO, USA) and electric coagulation. The appendiceal base was ligated by using one Endo loop (Ethicon, Somerville, NJ, USA). The specimen of appendix was removed through the Octoport wound retractor. W-SILS-A was performed using the same method was used for C-SILS-A, except that the former included wound infiltration with $10 \mathrm{mg}$ of $0.5 \%$ bupivacaine around the umbilicus just before the incision (Fig. 1).

In all patients, a patient-controlled analgesia (Accufusor, WooYoung Medical, Seoul, Korea) was used. As the patient-controlled analgesia, $18 \mu \mathrm{g} / \mathrm{kg}$ of fentanyl and $3 \mathrm{mg} / \mathrm{kg}$ of Keromin (Ketorolac Tromethamine) were diluted with metoclopramide and saline

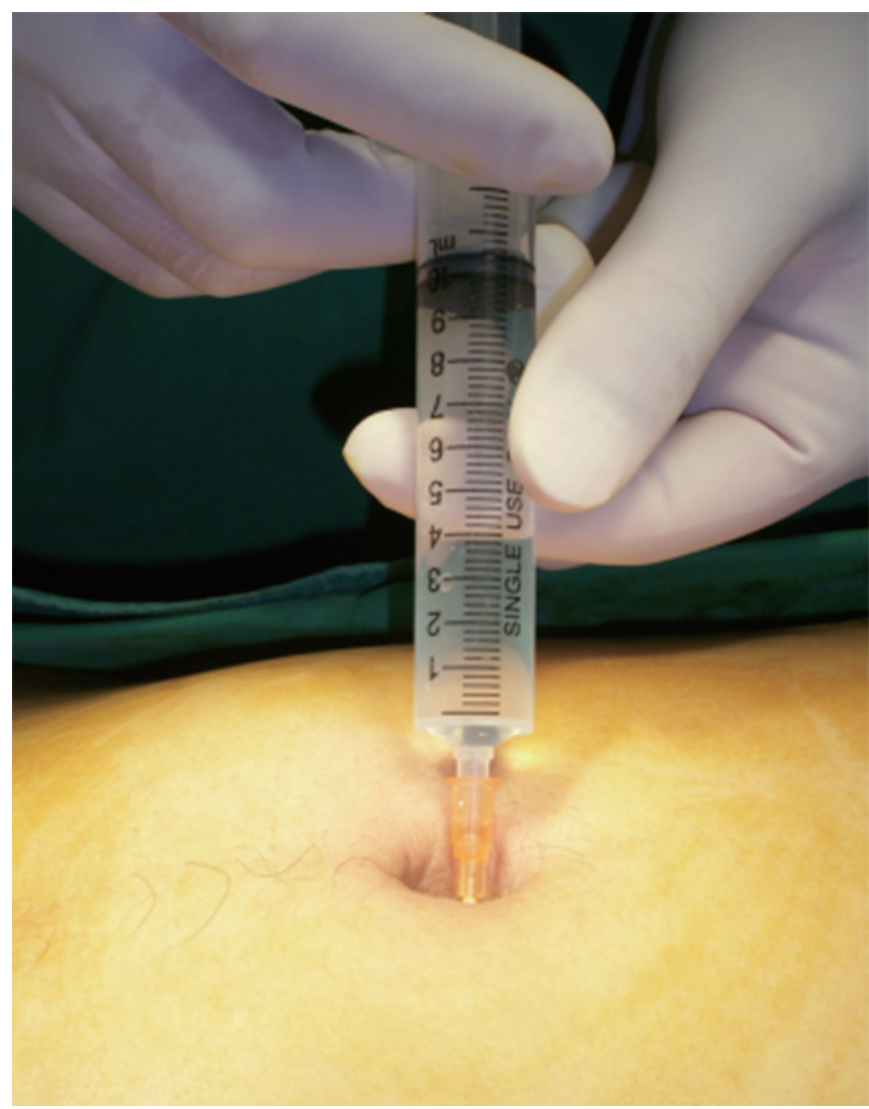

Fig. 1. Wound infiltration with $10 \mathrm{mg}$ of $0.5 \%$ bupivacaine around the umbilicus just before incision.

to a $100-\mathrm{mL}$ volume and injected. For cases presenting with severe pain, higher than 5 points on a verbal numerical rating scale (VNRS), despite the use of patient-controlled analgesia, an additional analgesic, Keromin (Ketorolac Tromethamine) was injected intravenously.

Statistical analyses were performed by using the Student t-test and the chi-square test with the SPSS ver. 17.0 (SPSS Inc., Chicago, IL, USA). P-values lower than 0.05 were considered to be statistically significant.

\section{RESULTS}

The ratio of males to females for the patients who underwent $\mathrm{C}$ SILS-A was 27:18, and their mean age was $23.7 \pm 14.5$ years. In the group that underwent a W-SILS-A, the ratio of males to females was 16:14, and their mean age was $36.0 \pm 13.2$ years. The operation time of the group that underwent C-SILS-A was $42.5 \pm$ 12.5 minutes, and that of the group that underwent W-SILS-A was $44.0 \pm 15.5$ minutes. Although the time was longer for the group that underwent W-SILS-A, the difference was not statistically significant $(\mathrm{P}=0.267)$.

As clinical courses, the time to the first gas out after surgery of 
Table 1. Demographic data and operative comparison between CSILS-A and W-SILS-A in appendicitis

\begin{tabular}{lccc}
\hline Variable & C-SILS-A $(\mathrm{n}=45)$ & W-SILS-A $(\mathrm{n}=30)$ & P-value \\
\hline Gender (male/female) & $27 / 18$ & $16 / 14$ & 0.252 \\
Age $(\mathrm{yr})$ & $38.5 \pm 14.5$ & $36.0 \pm 13.2$ & 0.624 \\
Body mass index $\left(\mathrm{kg} / \mathrm{m}^{2}\right)$ & $23.7 \pm 3.6$ & $23.2 \pm 3.2$ & 0.867 \\
Operation time (min) & $42.5 \pm 12.5$ & $44.0 \pm 15.5$ & 0.267 \\
Time until gas out (hr) & $18.0 \pm 6.5$ & $20.5 \pm 6.0$ & 0.250 \\
Time until diet start (hr) & $25.0 \pm 8.5$ & $27.5 \pm 9.5$ & 0.240 \\
Hospital stay (day) & $3.7 \pm 1.5$ & $3.4 \pm 1.0$ & 0.095 \\
No. of IV pain controls & $1.2 \pm 0.5$ & $0.2 \pm 0.2$ & 0.003 \\
\hline
\end{tabular}

Values are presented as mean \pm standard deviation.

C-SILS-A, conventional single-port laparoscopic surgery for an appendectomy; WSILS-A, wound infiltrated with $10 \mathrm{mg}$ of $0.5 \%$ bupivacaine in single-incision laparoscopic surgery for an appendectomy; IV, intravenous.

Table 2. Postoperative complications in C-SILS-A and W-SILS-A

\begin{tabular}{lcc}
\hline & C-SILS-A $(n=45)$ & W-SILS-A $(n=30)$ \\
\hline Wound seroma & 1 & 2 \\
lleus & 1 & 0 \\
Overall, $n(\%)$ & $2(4.4)$ & $2(6.6)$ \\
\hline
\end{tabular}

C-SILS-A, conventional single-port laparoscopic surgery for an appendectomy; $\mathrm{W}$ SILS-A, wound infiltrated with $10 \mathrm{mg}$ of $0.5 \%$ bupivacaine in single-incision laparoscopic surgery for an appendectomy.

the group that underwent C-SILS-A was $18.0 \pm 6.5$ hours, and that of the group that underwent W-SILS-A was $20.5 \pm 6.0$ hours. The times to the first diet after surgery were $25.0 \pm 8.5$ hours and $27.5 \pm$ 9.5 hours, and the hospitalization periods were $3.7 \pm 1.5$ days and $3.4 \pm 1.0$ days, respectively. The time to the first gas out after surgery, the time to the start of diet, and the hospitalization period showed no statistically significant differences.

The frequency of additional analgesics administered to the CSILS-A group was $1.2 \pm 0.5$ times, and that for the W-SILS-A group was $0.2 \pm 0.2$ times. The frequency of additional analgesics in the C-SILS-A group was higher than that in the W-SILS-A, the difference being statistically significant $(P=0.003)$ (Table 1$)$. In regard to postoperative complications, in the C-SILS-A group, seroma in the umbilical area developed in one patient, and ileus developed one patient. In the W-SILS-A group, seroma developed in two patients. They recovered after conservative management (Table 2). No patient in either group was converted to a laparotomy. Based on the VNRS, during the first postoperative 48 hours, the W-SILS-A group reported a significantly lower average VNRS score than the C-SILA-A group $(\mathrm{P}<0.05)$ during the first 1,6 , and 12 postoperative hours (Fig. 2).

\section{DISCUSSION}

As the interest in nonscarring surgical methods is on the rise with

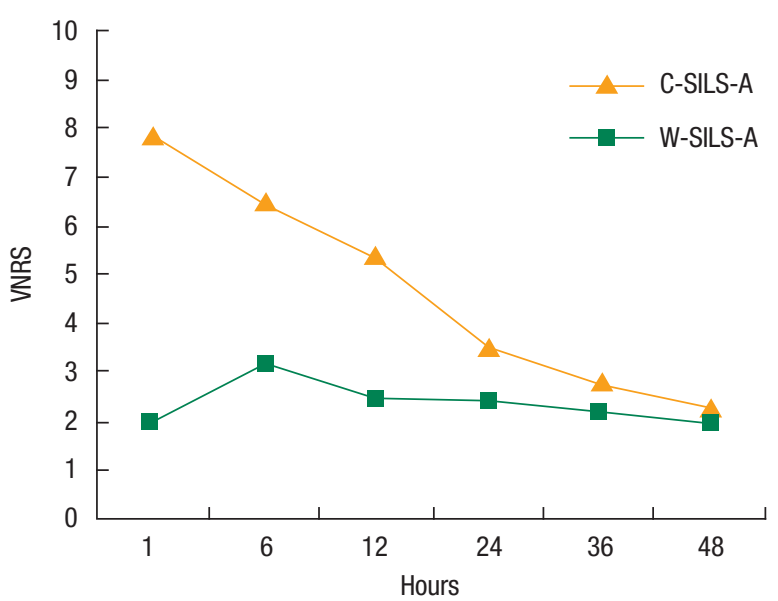

Fig. 2. Verbal numerical rating scale (VNRS) during the first postoperative 48 hours. SILS, single-incision laparoscopic surgery; CSILS-A, conventional SILS-A group; W-SILS-A, wound infiltrated with $0.5 \%$ bupivacaine in SILS-A group.

the development of equipment, laparoscopic minimally invasive surgery has increased in its use, such as in natural orifice transluminal endoscopic surgery and single-trocar or single-incision surgical methods, nowadays [3-8]. Usually, umbilical access is a well-known and standardized way to enter the abdominal cavity for laparoscopic minimally invasive surgery. Because the umbilicus is located in the middle of the abdomen, diverse intra-abdominal approaches can be performed. Blood vessels and nerves are absent, so incision windows can be readily created. Even after surgery, wounds become depressed within the umbilicus and, thus, may be considered as existing congenital scars [9-15]. Umbilical access is also applied to the SILS-A, which is a new technique of minimally invasive surgery.

Reviewing the reports that compared SILS-A with C-LA in adults, the former was found to be technically feasibility and to have good cosmetic results in addition to having the advantages of C-LA [9-13]. Nonetheless, some papers reported that SILS-A had a longer operation time and substantially early complaints of postsurgical pain [14-16]. In order to provide long postoperative analgesia and reduce intraoperative visceral pain, there have been many studies on the administration of both local anesthetics into the wound before the incision (preemptive analgesia) and opioids in various kinds of operation [17-22, 24]. A few studies investigated the effectiveness of wound infiltration with local anesthetics for postoperative pain relief in patients who underwent an open appendectomy, but the results remain controversial [25-28].

Ko et al. [25] used a combination of lidocaine hydrochloride and bupivacaine hydrochloride, and reported no benefits in reducing postoperative pain and analgesic requirements or in shortening the length of hospital stay, but their technique of anesthetics infiltration did not include the abdominal muscular layer, but used subcutaneous tissue. Lohsiriwat et al. [26] infiltrated $0.5 \%$ bupiva- 
caine into the muscular layer after skin and subcutaneous tissue had been incised and reported benefits in reducing postoperative pain during the first $6,12,24$, and 48 postoperative hours. Edwards et al. [27] used $0.25 \%$ bupivacaine following paediatric appendectomy and reported no additional benefit over regular simple analgesia, but their technique of wound infiltration did not include the abdominal muscle layer, but used the neurovascular plane and subcutaneous tissue prior to skin closure. In a study on C-LA, Cervini et al. [28] also used $0.5 \%$ bupivacaine and reported the benefit of preemptive bupivacaine infiltration, which resulted in a decreased need for postoperative parenteral narcotics.

A few studies have also assessed pain after SILS-A. Teoh et al. [14] reported in a prospective double-blinded randomized controlled trial no differences in the overall pain score and the pain score at rest between SILS-A and C-LA. However, patients in the SILS-A group experienced significantly more pain upon coughing or standing and required more intravenous analgesics. In another prospective study, Park et al. [15] reported no differences in the amounts of IV pain controls that were used during hospitalization between SILS-A and C-LA. However, the visual analogue scale pain scores during the first 24 postoperative hours were significantly higher in the patients who underwent SILS-A. Kim et al. [16] also reported the same results that the differences in the total doses of analgesics (nonsteroidal, anti-inflammatory drugs) administered during the 24 hours following surgery were not statistically significant, but the pain scores in the 24 hours after surgery were higher in patients who underwent SILS-A. This might be explained by the fact that the length of the fascial incision is closely associated with postoperative wound pain. Although the skin incision in the umbilical area is small, the actual length of the fascia incision is longer in SILS-A than it is in C-LA, and through the small incision window, laparoscopic equipment is used at once, which irritates the incision window. In our study, the WSILS-A group showed significantly lower numbers of additional pain killers and lower VNRS scores at 1, 6, and 12 hours after surgery than the C-SILS-A group.

Based on a theoretical basis, we thought that wound infiltration with $0.5 \%$ bupivacaine around the umbilicus would yield an analgesic effect by fascia anesthetic infiltration. The concept of preemptive analgesia is based on the hypothesis that the most effective way to eliminate or reduce postoperative pain is to prevent nociceptive input from afferent stimuli to the central nervous system so that central-nervous-system hyper-excitability does not occur [29]. Preemptive analgesia with a local anesthetic agent can be applied by using many methods, such as spinal blocks, epidural blocks, and preincisional infiltration. Several kinds local anesthetics, such as lidocaine, bupivacaine, levobupivacaine, and ropivacaine can be used for wound infiltration to provide postoperative pain relief.

Lidocaine is an amide local anesthetic that has many pharmacological features, such as antiarrhythmic, analgesic and anti-inflammatory properties. It has a rapid onset of action and an inter- mediate duration of efficacy. Its most adverse reactions as an anesthesia are related to the administration technique and result in central nervous system excitation and cardiovascular effects; allergic reactions rarely occur [30].

Bupivacaine is often administered by epidural injection during spinal anesthesia. It is most commonly injected into surgical wound sites for the relief of postoperative pain and is long-acting [19-21, 23, 25-28]. Systemic exposure to excessive quantities of it results in central nervous system excitation and cardiovascular effects, including hypotension, bradycardia, arrhythmias, and/or cardiac arrest [23].

Levobupivacaine is an S-isomer of racemic bupivacaine that has recently been introduced as a promising long-acting local anesthetic with a lower toxicity than bupivacaine [18]. Ropivacaine was developed after bupivacaine was noted to be associated with cardiac arrest, particularly in pregnant women [31]. Thus, bupivacaine has been superceded by levobupivacaine or ropivacaine, both of which have less propensity to produce cardiovascular depression and seizure activity because of overdose or intravascular injection, especially in neonates, children, or pregnant women $[18,31,32]$.

In our study, we used $0.5 \%$ bupivacaine as the local anesthetics for wound infiltration to provide postoperative pain relief. It is most commonly injected into surgical wound sites for the relief of postoperative pain, has a longer half-life than lidocaine, and can potentially provide relief of postoperative pain for up to 20 hours after the surgery, which were the reasons we selected it as the pain relief drug for wound infiltration [24].

In conclusion, this study shows that preincisional bupivacaine infiltration is an effective and simple method of reducing postoperative pain for patients undergoing SILS-A. Larger studies will be necessary to verify the true benefit of this method.

\section{CONFLICT OF INTEREST}

No potential conflict of interest relevant to this article was reported.

\section{ACKNOWLEDGMENTS}

This paper was supported by Wonkwang University 2011.

\section{REFERENCES}

1. Wei B, Qi CL, Chen TF, Zheng ZH, Huang JL, Hu BG, et al. Laparoscopic versus open appendectomy for acute appendicitis: a metaanalysis. Surg Endosc 2011;25:1199-208.

2. Tiwari MM, Reynoso JF, Tsang AW, Oleynikov D. Comparison of outcomes of laparoscopic and open appendectomy in management of uncomplicated and complicated appendicitis. Ann Surg 2011;254:927-32.

3. Trastulli S, Cirocchi R, Desiderio J, Guarino S, Santoro A, Parisi A, et al. Systematic review and meta-analysis of randomized clinical 
trials comparing single-incision versus conventional laparoscopic cholecystectomy. Br J Surg 2013;100:191-208.

4. White WM, Haber GP, Goel RK, Crouzet S, Stein RJ, Kaouk JH. Single-port urological surgery: single-center experience with the first 100 cases. Urology 2009;74:801-4.

5. Bucher P, Pugin F, Morel P. Single port access laparoscopic right hemicolectomy. Int J Colorectal Dis 2008;23:1013-6.

6. Oltmann SC, Rivas H, Varela E, Goova MT, Scott DJ. Single-incision laparoscopic surgery: case report of SILS adjustable gastric banding. Surg Obes Relat Dis 2009;5:362-4.

7. Huddy JR, Jamal K, Soon Y. Single port Billroth I gastrectomy. J Minim Access Surg 2013;9:87-90.

8. Chinnusamy P, Ahluwalia JS, Palanisamy S, Seshiyer RP. Single incision multi-trocar hepatic cyst excision with partial splenectomy. J Minim Access Surg 2013;9:91-4.

9. Kye BH, Lee J, Kim W, Kim D, Lee D. Comparative study between single-incision and three-port laparoscopic appendectomy: a prospective randomized trial. J Laparoendosc Adv Surg Tech A 2013;23:431-6.

10. Hong TH, Kim HL, Lee YS, Kim JJ, Lee KH, You YK, et al. Transumbilical single-port laparoscopic appendectomy (TUSPLA): scarless intracorporeal appendectomy. J Laparoendosc Adv Surg Tech A 2009;19:75-8.

11. Vidal O, Valentini M, Ginesta C, Marti J, Espert JJ, Benarroch G, et al. Laparoendoscopic single-site surgery appendectomy. Surg Endosc 2010;24:686-91.

12. Frutos MD, Abrisqueta J, Lujan J, Abellan I, Parrilla P. Randomized prospective study to compare laparoscopic appendectomy versus umbilical single-incision appendectomy. Ann Surg 2013; 257:413-8.

13. Rakkow R, Jacob DA. Initial experience in laparoscopic singleport appendectomy: a pilot study. Dig Surg 2011;28:74-9.

14. Teoh AY, Chiu PW, Wong TC, Poon MC, Wong SK, Leong HT, et al. A double-blinded randomized controlled trial of laparoendoscopic single-site access versus conventional 3-port appendectomy. Ann Surg 2012;256:909-14.

15. Park JH, Hyun KH, Park CH, Choi SY, Choi WH, Kim DJ, et al. Laparoscopic vs transumbilical single-port laparoscopic appendectomy; results of prospective randomized trial. J Korean Surg Soc 2010;78:213-8.

16. Kim HO, Yoo CH, Lee SR, Son BH, Park YL, Shin JH, et al. Pain after laparoscopic appendectomy: a comparison of transumbilical single-port and conventional laparoscopic surgery. J Korean Surg Soc 2012;82:172-8.

17. Hadj A, Hadj A, Hadj A, Rosenfeldt F, Nicholson D, Moodie J, et al. Safety and efficacy of extended-release bupivacaine local anaesthetic in open hernia repair: a randomized controlled trial. ANZ J Surg 2012;82:251-7.

18. Cantore F, Boni L, Di Giuseppe M, Giavarini L, Rovera F, Dionigi G. Pre-incision local infiltration with levobupivacaine reduces pain and analgesic consumption after laparoscopic cholecystectomy: a new device for day-case procedure. Int J Surg 2008;6 Suppl
1:S89-92.

19. Ozmen S, Ozmen OA, Kasapoglu F. Effects of levobupivacaine versus bupivacaine infiltration on postoperative analgesia in pediatric tonsillectomy patients: a randomized, double-blind, placebo-controlled study. Ann Otol Rhinol Laryngol 2011;120:489-93.

20. Kato J, Ogawa S, Katz J, Nagai H, Kashiwazaki M, Saeki H, et al. Effects of presurgical local infiltration of bupivacaine in the surgical field on postsurgical wound pain in laparoscopic gynecologic examinations: a possible preemptive analgesic effect. Clin J Pain 2000;16:12-7.

21. Hannibal K, Galatius H, Hansen A, Obel E, Ejlersen E. Preoperative wound infiltration with bupivacaine reduces early and late opioid requirement after hysterectomy. Anesth Analg 1996;83: 376-81.

22. Rikalainen-Salmi R, Forster JG, Makela K, Virolainen P, Leino KA, Pitkanen MT, et al. Local infiltration analgesia with levobupivacaine compared with intrathecal morphine in total hip arthroplasty patients. Acta Anaesthesiol Scand 2012;56:695-705.

23. Roberge CW, McEwen M. The effects of local anesthetics on postoperative pain. AORN J 1998;68:1003-12.

24. Parpaglioni R, Baldassini B, Barbati G, Celleno D. Adding sufentanil to levobupivacaine or ropivacaine intrathecal anaesthesia affects the minimum local anaesthetic dose required. Acta Anaesthesiol Scand 2009;53:1214-20.

25. Ko CY, Thompson JE Jr, Alcantara A, Hiyama D. Preemptive analgesia in patients undergoing appendectomy. Arch Surg 1997; 132:874-7.

26. Lohsiriwat V, Lert-akyamanee N, Rushatamukayanunt W. Efficacy of pre-incisional bupivacaine infiltration on postoperative pain relief after appendectomy: prospective double-blind randomized trial. World J Surg 2004;28:947-50.

27. Edwards TJ, Carty SJ, Carr AS, Lambert AW. Local anaesthetic wound infiltration following paediatric appendicectomy: a randomised controlled trial: Time to stop using local anaesthetic wound infiltration following paediatric appendicectomy? Int J Surg 2011;9:314-7.

28. Cervini P, Smith LC, Urbach DR. The effect of intraoperative bupivacaine administration on parenteral narcotic use after laparoscopic appendectomy. Surg Endosc 2002;16:1579-82.

29. Woolf CJ. Generation of acute pain: central mechanisms. Br Med Bull 1991;47:523-33.

30. Yardeni IZ, Beilin B, Mayburd E, Levinson Y, Bessler H. The effect of perioperative intravenous lidocaine on postoperative pain and immune function. Anesth Analg 2009;109:1464-9.

31. Gutton C, Bellefleur JP, Puppo S, Brunet J, Antonini F, Leone M, et al. Lidocaine versus ropivacaine for perineal infiltration postepisiotomy. Int J Gynaecol Obstet 2013;122:33-6.

32. Ozmen S, Ozmen OA, Kasapoglu F. Effects of levobupivacaine versus bupivacaine infiltration on postoperative analgesia in pediatric tonsillectomy patients: a randomized, double-blind, placebo-controlled study. Ann Otol Rhinol Laryngol 2011;120:489-93. 